\title{
A Generic Discrete-Event Simulation Model for Outpatient Clinics in a Large Public Hospital
}

\author{
Waressara Weerawat ${ }^{1 *}$, Juta Pichitlamken ${ }^{2}$ and Peerapong Subsombat ${ }^{1}$ \\ ${ }^{1}$ Department of Industrial Engineering, Mahidol University, Nakornpathom, Thailand \\ ${ }^{2}$ Department of Industrial Engineering, Kasetsart University, Bangkok, Thailand
}

Submitted November 2012. Accepted for publication February 2013.

\begin{abstract}
The orthopedic outpatient department (OPD) ward in a large Thai public hospital is modeled using Discrete-Event Stochastic (DES) simulation. Key Performance Indicators (KPIs) are used to measure effects across various clinical operations during different shifts throughout the day. By considering various KPIs such as wait times to see doctors, percentage of patients who can see a doctor within a target time frame, and the time that the last patient completes their doctor consultation, bottlenecks are identified and resource-critical clinics can be prioritized. The simulation model quantifies the chronic, high patient congestion that is prevalent amongst Thai public hospitals with very high patient-to-doctor ratios. Our model can be applied across five different OPD wards by modifying the model parameters. Throughout this work, we show how DES models can be used as decision-support tools for hospital management.
\end{abstract}

Keywords: hospital management, outpatient department, discrete-event stochastic simulation, healthcare simulation

\section{INTRODUCTION}

This work studies the orthopedic outpatient department (OPD) wards of a large public teaching hospital in Bangkok, Thailand. On average, the hospital serves 6,800 outpatients per day on weekdays and 1,800 outpatients per day during the weekends. The hospital is in the process of moving five wards (orthopedics, dermatology, otolaryngology, surgery, and medicine) out of 11 from the current location, which is highly congested and in disrepair, to a new building.

In this paper, our ultimate goal is to estimate the system capability and service level while quantifying the marginal impact of the new initiatives within the OPDs at the new hospital site. Besides some parameter changes, such as the number of staff or the patient arrival rates, the steps of outpatient flows remain the same in both settings; therefore, we will use a discrete-event simulation (DES) model of the as-is ward to assess performance at the new location.

*Corresponding Author: Waressara Weerawat, Logistics Innovation Center, Department of Industrial Engineering, Faculty of Engineering, Mahidol University, 999 Phuttamonthon 4 Rd., Salaya, Nakornpathom 73170, Thailand. Phone: (+662) 889-2138. Fax: (+662) 441-9731.

Email:waressara.wee@mahidol.ac.th. Other authors: juta.p@ku.ac.th,psubsombat@gmail.com. 
One of the attractive features of simulation modeling is its ability to model complex systems with random inputs (see [1,2] for the discrete-event simulation methodology). For example, in hospitals, patient arrival rates, patient types, treatment types, and treatment times are probabilistic. With computer models, what-if analysis and comparison of alternatives can be done without interfering with the real systems while generally using much less time and money to run experiments. These goals are common in most healthcare simulation publications.

Nevertheless, the implementation of simulation for operations improvement in healthcare is less widespread than in other industries. The hospital service sector is considered to be a secluded industry [3]. In particular, within the Thai public hospital setting, doctors or clinical experts often serve as the executive leaders, conducting a wide array of nonclinical operational activities such as accounting, IT, operations, and others. The Key Performance Indicators (KPIs) for non-clinical hospital operations have not been widely imposed by national health service associations such as the Thai Health Association (HA). They focus primarily on measuring clinical issues rather than OPD operations efficiency. The KPIs such as the average lengths of hospital stay for each inpatient visit, rate of unplanned readmission of the inpatient, and postoperative infection rate are examples of the clinical focused KPIs. Some service indicator measures such as satisfaction rates may be considered, but will be based mostly on opinion surveys of clinical service quality. In general, public hospitals receive major subsidy support from the government. The service prices at public hospitals can be $30-80 \%$ cheaper than those at private hospitals. Many patients such as government employees will be reimbursed for using the public hospital services. Other non-government employees entitled to social security or universal coverage receive service at designated hospitals which are mostly public ones. From the patients' perspective, using the public hospital service can be seen as a trade off between cheap care and convenience. In using private hospitals, most patients need to pay out of their own pockets or use private insurance. With greater income, private hospitals create higher demand for their respective target customers by attracting well-known doctors, getting contract services, and offering higher levels of customer service.

In this paper, we propose various KPIs to be used for non-clinical operations' improvement. In our example, some of the proposed alternatives have already been implemented by executives in large public hospitals without formal measuring tools for non-clinical purposes. We recommend the proper KPIs and show a concrete example of using simulation as a tool to compare and quantify the marginal benefits of the proposed alternatives. This helps in justifying the trade-offs for investment in the Thai public hospital sector which traditionally focuses primarily on patient safety.

Seila and Brailsford discuss opportunities and challenges in applying DES and system dynamics (SD) simulation to healthcare [3]. Their survey paper begins by describing similarities and differences across healthcare systems. DES and SD simulations are described and compared using healthcare applications. They classify healthcare models into three groups: Level 1 models consider the human body; Level 2 models cover operational or tactical levels, such as patient's movements within the system; and Level 3 is strategic, system-wide models that do not consider patients 
individually. DES is mostly chosen to construct Levels 1-2 models whereas SD is preferred for Level 3. Our model belongs to Level 2, covering operational and tactical levels with a focus on patients' movements within the OPD.

Weng and Houshmand model an outpatient clinic using Arena software which is one of the DES commercial packages [4]. Their goal is to compare different resident staffing scenarios in terms of cost, patient throughput and the total time in system. Similarly, Raouf and Ben-Daya consider the number of doctors for an outpatient clinic in Saudi Arabia such that the patient waiting time is within a pre-specified target [5]. In addition to the staffing problem, many simulation studies [6-8] compare appointment scheduling configurations with respect to performance measures that consider both customer satisfaction (such as the average length of queue, and patient waiting time) and system efficiency, e.g., doctor's utilization and overtime.

Simulation models are also developed for process improvement or for determining the process bottlenecks and system capacity. Ballard and Kuhl model the surgical suite operating room using the DES commercial software Arena to determine its daily maximum capacity, defined as the number of patients treated [9]. They show that the capacity estimated by simulation is higher than what the management has calculated through considering only the ratio of available times and the required treatment time, and thus higher efficiency of the operating rooms can be realized. Beasler et al. consider a similar problem, but for emergency rooms [10]. Werker et al. aim to study the process improvement of radiation therapy treatment [11], while Duguay and Chetouane [12] and Brenner et al. [13] consider emergency departments. Raghavan et al. adopt a lean approach for process improvement at cardiac catheterization labs [14].

Related to process improvement, simulation models are often used to determine the best operational settings, i.e., for optimization purposes. Oddoye et al. integrate their DES model with goal programming (one of the techniques for solving multiobjective optimization problems) to determine bottlenecks in clinical workflow and determine the optimal number of beds for different types of patients [15]. Villamizar et al. use MedModel software to model a physiotherapy clinic, and their model is further applied to select the optimal operational strategy (patient schedules and the number of staff) in terms of patient throughput rates and patient waiting times [16]. $\mathrm{Su}$ et al. also build their simulation models using MedModel to redesign a hospital registration process [17].

In this paper, we develop a simulation model for an orthopedic ward which serves approximately 2,600 patients per week. Through on-site observations and interviews with the hospital staff, especially the head nurses, at all five wards that will be relocated, we find that the patient flows are mostly similar; they differ only in details such as number and types of patients, number of staff, or office hours of special clinics. These common features allow us to construct a conceptual flow of outpatients as shown in Figure 1.

The OPD process for a by-appointment patient begins with presenting the appointment card at a registration desk. If a patient is scheduled for a blood test or an $\mathrm{X}$-ray test, it must be done before vital sign checks which include taking measurements of temperature, respiratory rate, pulse rate and blood pressure. Then a doctor queue card 




Figure 1. Patient flow of the orthopedic ward. 
is issued. After consulting with a doctor, a patient waits for an appointment card for the next visit and any prescription. Then, payment is made and medication received at one of the in-house pharmacies.

Patients without appointments (walk-in and transferred) also arrive at the OPD registration desk; however, if all doctor's time slots are filled, they will be scheduled for visits on later days. Some patients arrive without appointments because they know that OPDs sometimes accept walk-in patients. Two different criteria are used to determine the admission of the non-appointment patients depending upon their arrival time. The first criterion is the expected remaining treatment slots in each shift (Module D07 in Figure 1), which applies in general. However, if a patient arrives within an hour of the shift end time, the modified second criterion which accounts for the remaining patients in the clinic (work in process, WIP) at the patient arrival time will be applied (Module D08 in Figure 1).

Besides data collection and data cleaning, the major challenge in the model construction is to compute statistics for KPIs. Because the OPD is highly congested, doctors in the real OPD often work past their finish time (e.g., during lunch break) as specified on schedules. Our model captures this fact by allowing doctors to do "overtime." The duration of this overtime reflects the congestion in a ward; hospital management can use this simulation output to prioritize where service improvements are needed. Another facet of human behavior included in our model are the rules that head nurses employ to accept walk-in patients into appointment schedules. This decision is routinely made by intuition, and we establish concretely how it is done (Modules D06-08 in Figure 1).

After our simulation model is validated, we perform what-if analysis to experiment with our proposed improvement alternatives and the future scenario of increased number of patients. In the analysis, we illustrate how different KPIs can complement one another, and how they quantitatively reflect the actual performances of clinics.

The contributions of our work are (a) accumulating statistics for KPIs, with some of which complicated by human behaviors that try to accommodate high system congestion; (b) discussion of challenges in simulation model construction and our solutions; and (c) demonstrating that different KPIs can complement one another and shed light on the performance of clinics.

\section{METHODOLOGY}

\subsection{Input Modeling}

The schedule of the orthopedic ward is divided based on ten types of specialized clinics: general practice (A), children (B), tumor (C), off-hour (D), sport injuries (E), osteoporosis $(\mathrm{F})$, hands $(\mathrm{G})$, backs $(\mathrm{H})$, hips and knees (I), and special consultation (J). Table 1 shows the office hours of each ward. Some clinics are only open for limited time during the week; e.g., the children's clinic is only open on Monday afternoons. Many clinics may be available simultaneously; e.g., both the sports injuries (E) and osteoporosis (F) clinics are scheduled on Tuesday afternoons, and the percentages of patients for both clinics are parenthesized. The office hours of clinics affect the patient 
Table 1. The orthopedic OPD operation schedule

\begin{tabular}{lccccccc}
\hline Shift & Mon & Tue & Wed & Thu & Fri & Sat & Sun \\
\hline 1 (Morning) & A & A & A & A & A & D & D \\
2 (Afternoon) & B $(64 \%)$, & F $(7 \%)$, & G $(73 \%)$, & J & I & D & - \\
3 (Evening) & C $(36 \%)$ & E $(93 \%)$ & H $(27 \%)$ & & & & \\
& D & D & D & D & D & - & -
\end{tabular}

arrival rates. Patient arrival rates depend on operating shifts (morning, afternoon and evening), days of the week, and time of the day.

Table 2 exhibits the total number of patient arrivals by shifts and days of the week, and Table A1 (see APPENDIX) presents the weekly distribution of patient arrivals by patient types and shifts. These data allow us to model the patient arrival with the nonstationary Poisson Process [18]. Both in the actual system and in simulation, appointment and walk-in patients arrive from 7.00 to 18.00 hours (to only 14.00 on weekends), and transferred patients enter the ward from 10.00 to 18.00 (14.00 on weekends). The resources' schedule information of doctors, residents, and nurses is displayed in Table A2. The three types of patients (by-appointment, walk-in, and transferred) are given another attribute: either "simple" or "complicated" which denotes additional x-ray or blood tests required.

Besides interviews and paper/electronic records, our numerical data were collected from a stop-watch time study conducted during June 2010. The service time measures the length of time when service begins until it ends, and it does not include the waiting time before service begins. In fact, waiting time is part of our simulation output. We model the service time at different stages of patient care with the triangular distribution because it allows us to specify the minimum, maximum and most likely values when raw data are limited (sample size of 10-15 for each input process). When the sample size is small, the goodness-of-fit tests (e.g., Chisquare or Kolmogorov-Smirnov) tend to accept any probability distributions, whereas when the sample size is large, most distributions are rejected [19]. Thus, we choose the same probability distribution for all input data based on physical conditions. Details of our service times and their associated resources of some service processes are shown in Table 3.

Table 2. Patient arrival numbers of the orthopedic OPD

\begin{tabular}{lcccccccc}
\hline Patient Arrival & Mon & Tue & Wed & Thu & Fri & Sat & Sun & $\begin{array}{c}\text { Sum } \\
\text { (Weekly) }\end{array}$ \\
\hline 1 (Morning) & 223 & 244 & 244 & 190 & 280 & 260 & 97 & 1538 \\
2 (Afternoon) & 96 & 132 & 156 & 108 & 149 & 105 & 0 & 747 \\
3 (Evening) & 63 & 70 & 68 & 38 & 83 & 0 & 0 & 321 \\
Total & 382 & 446 & 468 & 336 & 512 & 365 & 97 & 2606
\end{tabular}


Table 3. Resources for service processes and service time parameters (module numbers correspond to Figure 1)

\begin{tabular}{lllcc}
\hline ID & \multicolumn{1}{c}{ Process } & Resource & $\begin{array}{c}\text { Service time parameters } \\
\text { (Min, Mode, Max) }\end{array}$ & Time Unit \\
\hline C01 & Present an appointment card & Nurse & $(4,5,7)$ & Second \\
C02 & Weigh and check vital signs & Weight center & $(30,45,60)$ & Second \\
C03 & Receive a queue card & Nurse & $(3,8,15)$ & Second \\
C04 & See a doctor & Doctor & $(2,12,25)$ & Minute \\
C05 & Receive injection & Nurse/Doctor & $(5,10,15)$ & Second \\
C06 & Receive diagnosis & Doctor & $(5,15,20)$ & Minute \\
C07 & Return medical record & Clerk & $(3,4,5)$ & Second \\
C08 & Receive appointment card & Nurse & $(5,23,45)$ & Second \\
C09 & Pay and receive medicine & Pharmacist & $(30,59,105)$ & Second \\
C10 & Receive blood test & Technician & $(2,2.5,3)$ & Minute \\
C11 & Receive X-ray & Technician & $(2,9,20)$ & Minute
\end{tabular}

\subsection{Simulation Model Construction}

We develop our simulation model using Arena (Rockwell Automation) which is a widely-used example of DES software. Arena uses a process view where an Entity is created and pushed into the system. A model is constructed by connecting built-in modules to form a flowchart through which an Entity flows and triggers events [18]. We have three types of patients as the Entities: By Appointment, Walk In and Transferred. They are further divided in terms of Attributes, such as Patient Number, Arrival Time, Simple (yes/no), Blood Test (yes/no), X Ray Test (yes/no), among others. Figure 1 shows the flow of patients in our simulation model which consists of clinical activities (C) modules and decision (D) modules. C-modules correspond to processes physically occur in the wards such as registration, vital sign checks, preparation of medical records, labs and $\mathrm{x}$-ray, physician consultation, next-appointment scheduling, cashier, and pharmacies, while D-modules are logics for decisions.

The simulation model is designed such that it can represent all five OPD wards by merely changing parameters such as processing time, proportion of patient types (appointment or non-appointment), percentage of patients requiring additional testing, numbers of clinical doctors, residents and nurses, and percentage of patients using three pharmacies. Table 4 shows examples of ward-specific probabilities of decision modules. Having a single model is appealing because an analyst needs to maintain only one model.

Due to the complexities of the hospital service which involves specialized treatments for individual patients, large variations of output performances are not surprising. Our simulation model returns the following KPIs:

- Total time that a patient is in the hospital: time duration since a patient enters the registration process until receiving a prescription and exiting the OPD.

- Patient waiting time: duration of time in queue, waiting to be called and excluding service time. 
Table 4. Details of decision modules for different OPDs (module numbers correspond to Figure 1)

\begin{tabular}{|c|c|c|c|c|c|c|}
\hline \multirow{2}{*}{$\begin{array}{l}\text { Module } \\
\#\end{array}$} & \multirow{2}{*}{$\begin{array}{l}\text { Name of decision } \\
\text { module }\end{array}$} & \multicolumn{5}{|c|}{ Outpatient Department (OPD) } \\
\hline & & Orthopedics & Skin 0 & Otolaryngology & Surgery & Medical \\
\hline D01 & $\begin{array}{l}\text { Require additional } \\
\text { testing? (Blood Test, } \\
\text { X-ray) }\end{array}$ & $(5 \%, 2 \%)$ & $(2 \%, 1 \%)$ & $(8 \%, 4 \%)$ & $(7 \%, 15 \%)$ & $(60 \%, 10 \%)$ \\
\hline D02 & $\begin{array}{l}\text { Require other } \\
\text { diagnosis? (Blood } \\
\text { Test, X-ray) }\end{array}$ & $(5 \%, 2 \%)$ & $(1 \%, 1 \%)$ & $(2 \%, 1 \%)$ & $(2 \%, 5 \%)$ & $(15 \%, 2 \%)$ \\
\hline D03 & $\begin{array}{l}\text { Require } \\
\text { injection? }\end{array}$ & Yes $(30 \%)$ & Yes $(40 \%)$ & Yes $(2 \%)$ & No & Yes $(5 \%)$ \\
\hline D04 & Require surgery? & Yes $(5 \%)$ & No & No & Yes $(10 \%)$ & No \\
\hline D05 & $\begin{array}{l}\text { Proceed to } \\
\text { General Practitioner } \\
\text { (GP) pharmacy? } \\
\text { (GP, Central, ER) }\end{array}$ & $\begin{array}{c}(90 \%, 5 \%, \\
5 \%)\end{array}$ & $\begin{array}{c}(0 \%, 100 \% \\
0 \%)\end{array}$ & $\begin{array}{c}(0 \%, 0 \% \\
100 \%)\end{array}$ & $\begin{array}{c}(0 \%, 100 \%, \\
0 \%)\end{array}$ & $\begin{array}{c}(0 \%, 100 \% \\
0 \%)\end{array}$ \\
\hline
\end{tabular}

- Time when the last patient finishes the doctor consultation by shift. This is important for determining the time duration that doctors spend beyond their scheduled office hours. It indicates if the current office hours can accommodate patient demand.

- Time-averaged number of patients in each clinic and in the overall OPD.

- Average utilization of chairs in the waiting area of the wards and at the pharmacies: percentage of time that they are occupied.

- Resource utilization of supporting staff and doctors: ratio of busy time to total time.

- Patient service levels with respect to time:

- Percentage of by-appointment patients who see doctors within 30 minutes after their arrivals.

- Percentage of walk-in and transferred patients who see doctors within 1 hour after their arrivals.

- Percentage of by-appointment patients who spend the total time in the system for less than 2 hours after their arrivals.

- Percentage of walk-in and transferred patients who spend the total time in the system for less than 3 hours after their arrivals.

Since the level of hospital services vary widely, the percentage of measures delivered within the target range as well as the minimum and maximum values should be considered in addition to the average values.

\subsection{Challenges in Data Collection and Model Construction}

The process of developing a simulation model is insightful in itself because we have to understand the actual process flow and collect quantitative data from the real system. Similar to many public tertiary hospitals in Thailand, our orthopedic ward 
serves a large number of patients; therefore, its doctors and nurses are so busy and the facility so crowded that allow us little time for data collection or interviews. Unlike at private hospitals, patients arrive at this hospital much earlier than the beginning of the office hours to get a queue card; some of them arrive 2 hours before the beginning of doctors' hours at 9 AM. The number of patient arrivals depends on the clinic office hours but not so much on the area of expertise of the doctor. In contrast, in private hospitals, the number of patients is dependent on the doctor's reputation.

Prior to fitting probability distributions for input modeling, we check for input inconsistency and perform data cleanup; for example, if the time log indicates that a patient arrives when the ward is actually closed, this data point will be discarded. The time logs of when patients first register are ideal for estimating the patient arrival rates. Instead, we only have the hospital's electronic time logs showing the times that patients are ready to see doctors. However, prior to meeting doctors, some patients may need medical scans or tests. We have to adjust the available time log data by considering the ratio of patients who need prior tests (and their respective time durations) and those who do not. In addition, the electronic time log does not specify if a patient is by appointment or a walk-in. After we manually determine the patients' arrival times and types, we check data validity with the domain experts.

The OPD wards are managed independently. We consider their interaction through the ratio of patients using shared facilities for blood and x-ray testing, and pharmacies. The main interactions are between special clinics within wards and not across the wards. Dependence between OPD wards is treated exogenously. For example, transferred patients include patients from other wards.

Another challenge in model construction is to implement our own statistical collections in Arena to report KPIs that are averages over multiple criteria: time duration (hourly, daily, or weekly), clinic type, patient type (walk-in, by appointment, or transferred), patient arrival, and doctor's round (Shift 1, Shift 2, or Shift 3). Many simulation outputs are time averages, e.g., resource utilization, number of patients in the waiting area, or number of patients in the OPD. Suppose we consider doctor utilization. Since doctors see a large number of patients daily, their actual finish times regularly exceed their scheduled office hours (this "overtime" is assumed to be $>1$ hour for utilization calculation). We specify the simulation run time to be 24 hours; therefore, resource schedules must be 24 hours long as well. Assume Arena returns doctor utilization rate of $\rho_{\text {Arena }}$ defined as the integration (area underneath the plot) of the number of busy doctors (seeing patients) $B(t)$ divided by the number of scheduled doctors $B(t)$ with respect to time $t$ :

$$
\rho_{\text {Arena }}=\frac{1}{24} \int_{0}^{24} \frac{B(t)}{S(t)} d t
$$

Let the actual operating time duration of clinic be $T$. Because the area under the curve $\int_{0}^{24}(B(t) / S(t)) d t$ remains the same, actual utilization is adjusted to 
Table 5. Two-sample t-test with unequal variance between the actual total time and the simulated total time

\begin{tabular}{lcc}
\hline & Empirical results & Simulation \\
\hline Mean (min) & 124.8 & 117.6 \\
Variance & 6803.9 & 7157.7 \\
Standard Deviation & 82.49 & 84.60 \\
Number of observations (patients) & 200 & 200 \\
Hypothesized mean difference & 0 & \\
Degree of freedom & 398 & \\
t Stat & 0.859 & \\
Prob $(\mathrm{T} \leq \mathrm{t})$ two-tail & 0.391 & \\
t Critical two-tail & 1.966 &
\end{tabular}

$$
\rho=\rho_{\text {Arena }} \frac{24}{T} \text {. }
$$

Other time-average outputs from Arena are adjusted in a similar manner.

\subsection{Model Validation}

We validate our simulation model via a two-sample t-test with unequal variance [19] between the actual total time in the OPD and the simulated total time. Let the population mean of the actual total time and the simulated value be $\mu_{1}$ and $\mu_{2}$, respectively. The twosample t-test considers the null hypothesis $\left(\mathrm{H}_{0}\right): \mu_{1}$ is equal $\mu_{2}$. Table 5 displays the daily averages of simulated patients' total time in the hospital based on the randomly selected values of 200 patients from a week-long (Monday through Saturday) simulation run. Similarly, the empirical data displayed in Table 5 is randomly selected from 200 different patients in one week. The two-sample t-test is employed to test the hypothesis, and the p-value of the two-sided test is 0.391; therefore, there is no evidence to disregard the validity of our simulation model compared with the actual system. Since there are limited data in the hospital settings, the validation process requires additional confirmation from the respective domain experts beyond the statistical testing, and the experts consider the KPIs estimated from our simulations reasonable.

\section{RESULTS AND DISCUSSION}

We first present the analysis of the as-is orthopedic ward in Section 3.1. Then we illustrate how the simulation model allows us to assess the impacts of process changes on service performance (Section 3.2). Our simulation model is naturally terminating because each day begins when no patients are in the system, and all resources are idle. The simulation run setup is: simulation run length is one week, and the number of replications is 30, resulting in a run time of approximately 12 minutes on a standard Intel Core i3 notebook PC with 4 GB installed memory (RAM). 


\subsection{The Present OPD}

The simulation outputs quantify and confirm patients' comments that they spend most of their times in the ward waiting for doctors; the average total time in the ward is $124.9 \pm 1.2$ minutes, most of which is the waiting time in queue (103.7 \pm 1.2 minutes) or $83 \%$ of the total time. This implies that the current value-added service to the patient is less than $20 \%$ which is relatively low.

Figure 2 shows the total time distribution. The first part (Modules C01 to C03, C10 and $\mathrm{C} 11$ in Figure 1) includes the steps from checking in until receiving the doctor queue card; the second part (Modules $\mathrm{C} 04$ to $\mathrm{C} 08$ ) covers the steps from being ready to see the doctor to the step of getting the next appointment; the third part (Module C09) considers the steps after receiving the future appointment to the step of receiving the medications outside the orthopedics ward. For all types of patients, waiting to see a doctor accounts for the major part of time expended.

The average number of patients in Figure 3 takes into account only those in the OPD waiting area. The long waits result in congestion in the waiting area and thus require more seats; the recommended number of seats is approximately twice the number of those currently available. As expected, these long delays are caused by a large number of patients compared to the number of available doctors (over some periods of times but not all the time). From Figure 3, we can see that the most congested days of the week are Tuesdays and Fridays because hips and knees and sports injuries clinics are

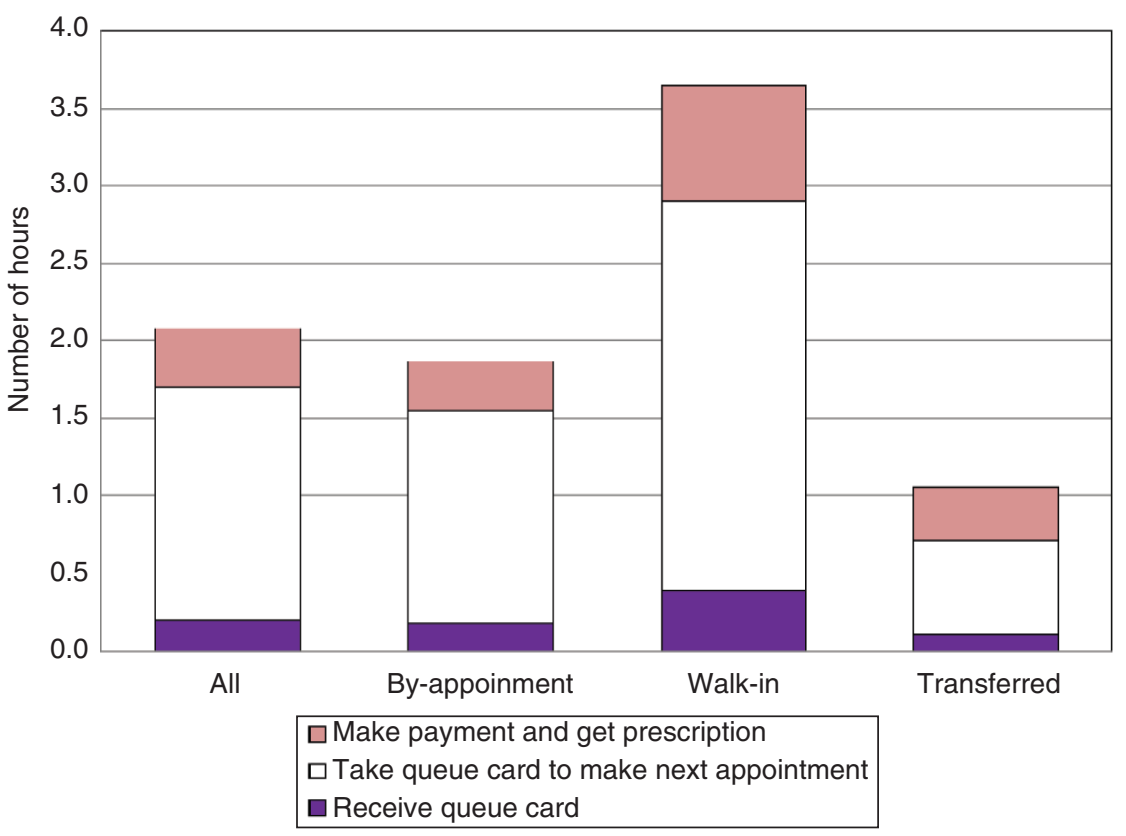

Figure 2. Distribution of time spent in the hospital by patient types. 


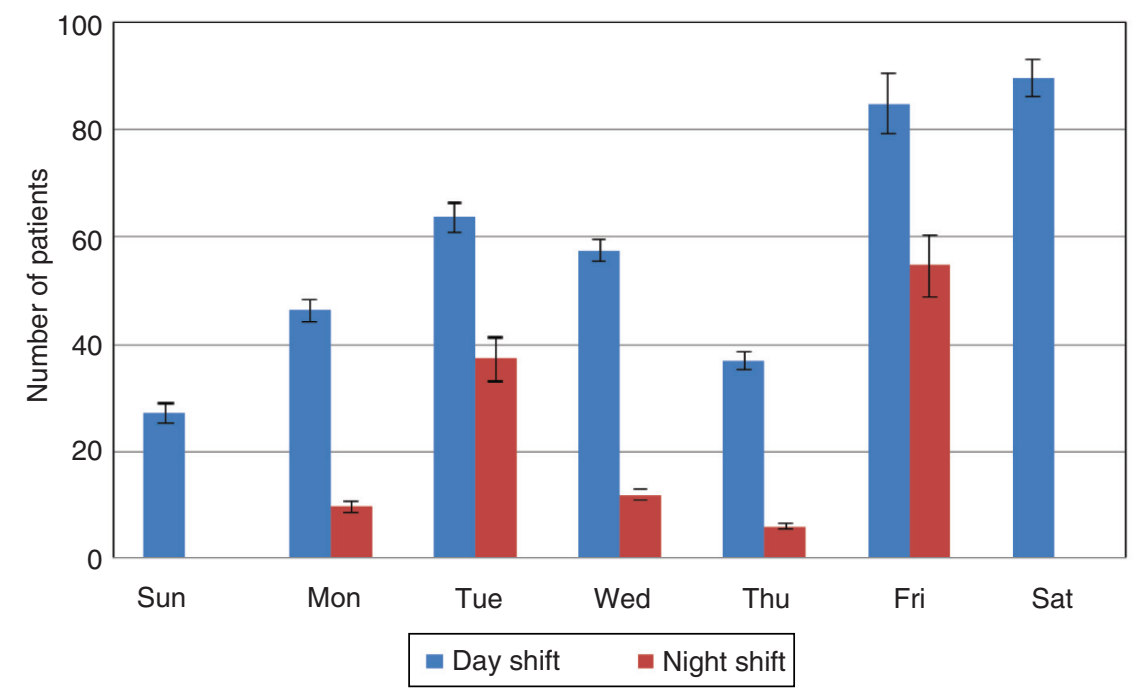

Figure 3. Average numbers of waiting patients in the OPD area.

scheduled on those days. Saturdays also see a large number of waiting patients because it is the off-hour clinic where the number of available doctors is small.

In terms of patient service levels, we can determine the percentage of patients who can either see the doctor or spend time in the hospital within a pre-determined target time frame as suggested in section 2.2. Simulation outputs show that $60 \%$ of the by-appointment patients wait for longer than 30 minutes, whereas $80 \%$ of the walk-in patients and $10 \%$ of the transferred patients wait for more than one hour. Similarly, when we consider the total time spent in the hospital, $38 \%$ of the byappointment patients wait for longer than 2 hours, while $71 \%$ of the walk-in patients and $3 \%$ of the transferred patients are there for longer than 3 hours. (The graphical display of these results can be viewed in Figure 7 by subtracting the blue bar values from $100 \%)$.

Figure 4 shows data of doctor utilization rate (eqn. 1,2) which reflect high patientto-doctor ratios in most clinics except the osteoporosis clinic (F). By adding one additional doctors' working hour beyond the normal operating hours for some clinics (see Tables A1-A2 in the APPENDIX), doctor utilization rate is still extremely high. Figure 4 also presents the utilization rates for different shifts of the off-hour clinic (D). The relatively low utilization rate of the off-hour clinic (D) on weekdays is due to the fact that this clinic generally does not have very high patient-to-doctor ratios. No patient is admitted during the last hour of operation. In actual hospital practice, doctors usually do not leave until all scheduled patients have been seen, even if working overtime. The doctors' overtime data in Figure 4 show that clinics with higher utilization rate tend to have longer doctors' overtime. Such data can support the decision concerning which 


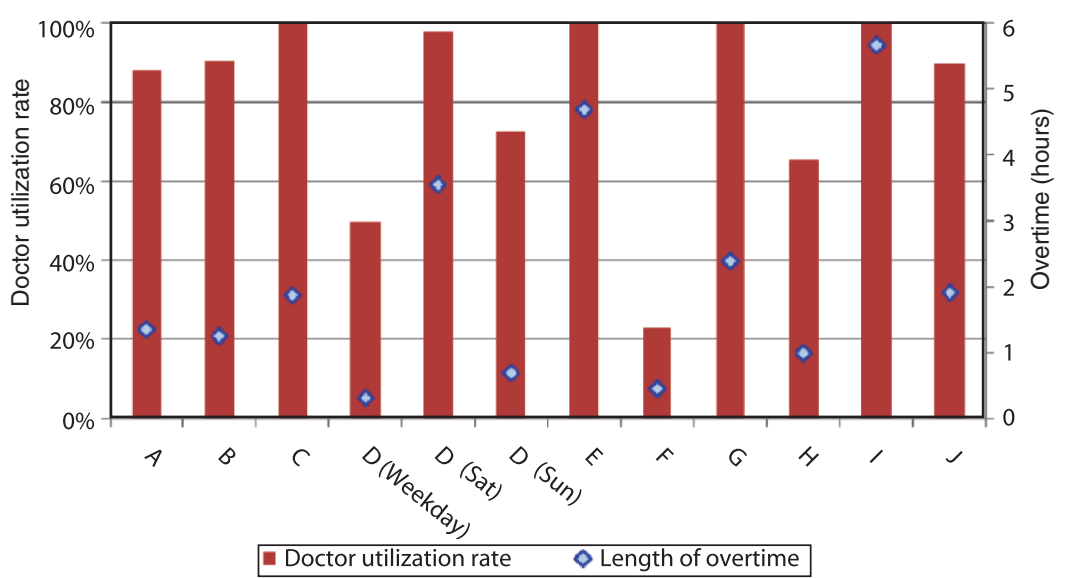

Figure 4. Doctor utilization rate and overtime by clinic.

clinic deserves adding more doctors and/or operating hours. Apparently, Clinic I should have the first priority because it has the highest utilization rate $(100 \%)$ and the longest doctor's overtime hours.

The present simulation results indicate low nurse utilization rate (data not shown) which reflects the relatively short time they serve patients in OPD. However, in reality, nurses are occupied all the time; perhaps they spend much time on activities or tasks not directly related to serving patients.

The present results indicate that the orthopedic ward does not fare very well in terms of long patients' waiting time. The situation will worsen if more patients need to be taken care of by the new hospital facility as the hospital has predicted. Possible improvements are proposed in the following sections.

\subsection{Proposed Measures to Improve the Present OPD}

Simulation models can be employed to predict impacts or effectiveness of process improvement plans without interfering with the real systems.

\subsubsection{Application of Information Technology}

Currently, medical records in OPD are paper based. They are made ready one day in advance of patients' appointments; however, about 20-30\% of the records are misfiled and need approximately 30 minutes per patient to be located when the patient checks in. If the electronic records are available, the retrieval times may be reduced to merely 30-90 seconds. Electronic records can also reduce paper handling time and possibility of lost records. With the proposed electronic system, a patient can directly go to the pharmacy after seeing the doctor. Figure 5 compares the number of patients waiting in the OPD waiting area with and without the proposed electronic medical record system, and the data show statistically significant decreases in the number of patients waiting on every day of the week. 


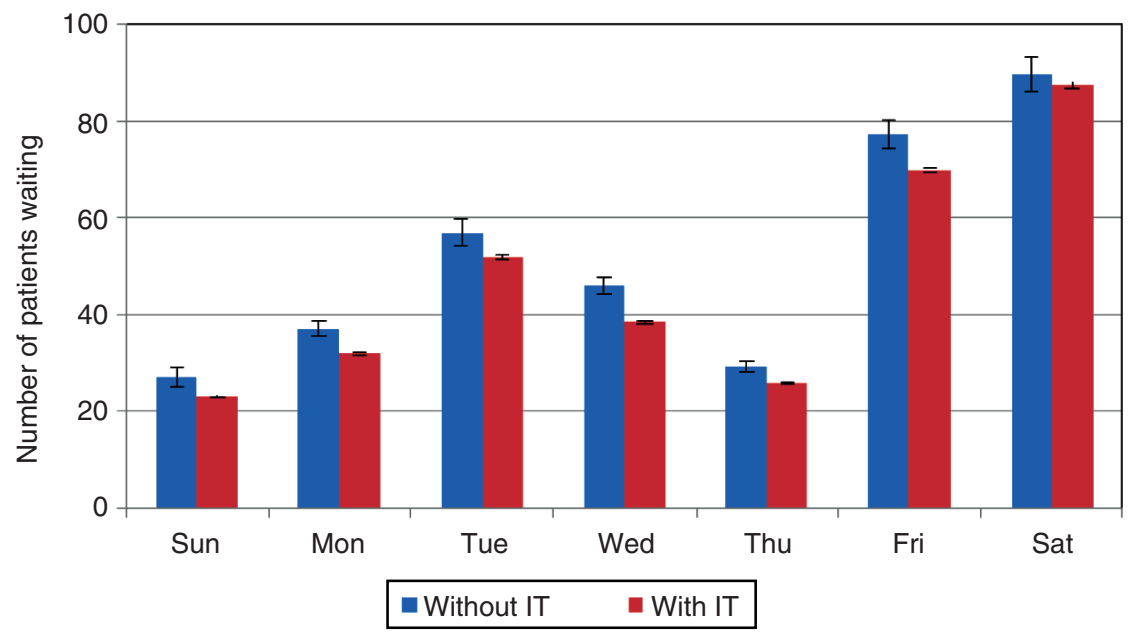

Figure 5. Comparison of the overall average number of patients in the OPD waiting area with and without the proposed electronic medical record (IT) system. Each error bar indicates the $95 \%$ confidence interval.

\subsubsection{Resource Schedule Changes}

Because of the very high patient-to-doctor ratio (see the last column of Table A3), waiting time will decrease drastically if the number of doctors increases, especially in the highly congested clinics such as hips and knees (I) and sports injuries (E). Based on the doctor utilization rate data in Figure 4, we can categorize our clinics into three groups. The most congested group includes tumor (C), sport injuries (E), hands $(\mathrm{G})$, and hips \& knees (I) clinics where the doctor utilization rate is more than $97 \%$. The second group includes general practice (A), children (B), off-hour (D) on Saturday, and special consultation $(\mathrm{J})$ clinics, where the doctor utilization rate is between 88-92\%. The third group includes off-hour (D) on weekdays and Sunday, osteoporosis $(\mathrm{F})$ and backs $(\mathrm{H})$ clinics. These clinics can accept more patients since their utilization rate is less than $75 \%$.

In general, if the overtime exceeds one hour, simulation experiment is conducted with increasing the number of doctors or rearranging the doctor's schedules. Currently, the morning schedule is from 9-12 AM (see Table A3 in appendix). We propose the straddled schedule which uses the same doctor-hours by having 4 doctors in the morning during 7-10, 4 doctors $8-11$ and another 3 9-12 noon (see Table A4 in APPENDIX). The rationale is that patients begin to arrive at 7 AM (partly due to the heavy city traffic constraints), and if doctors arrive earlier, resource usage can be more evenly spread throughout the morning and spanning over longer time. Under this proposal, the average total time a patient spends in the system reduces from $124.9 \pm 1.2$ minutes (11 doctors, 9-12 AM) to $48.6 \pm 9.6$ minutes (11 doctors, straddled). Figure 6 compares the average total time that a patient spends in the clinic before seeing a doctor 


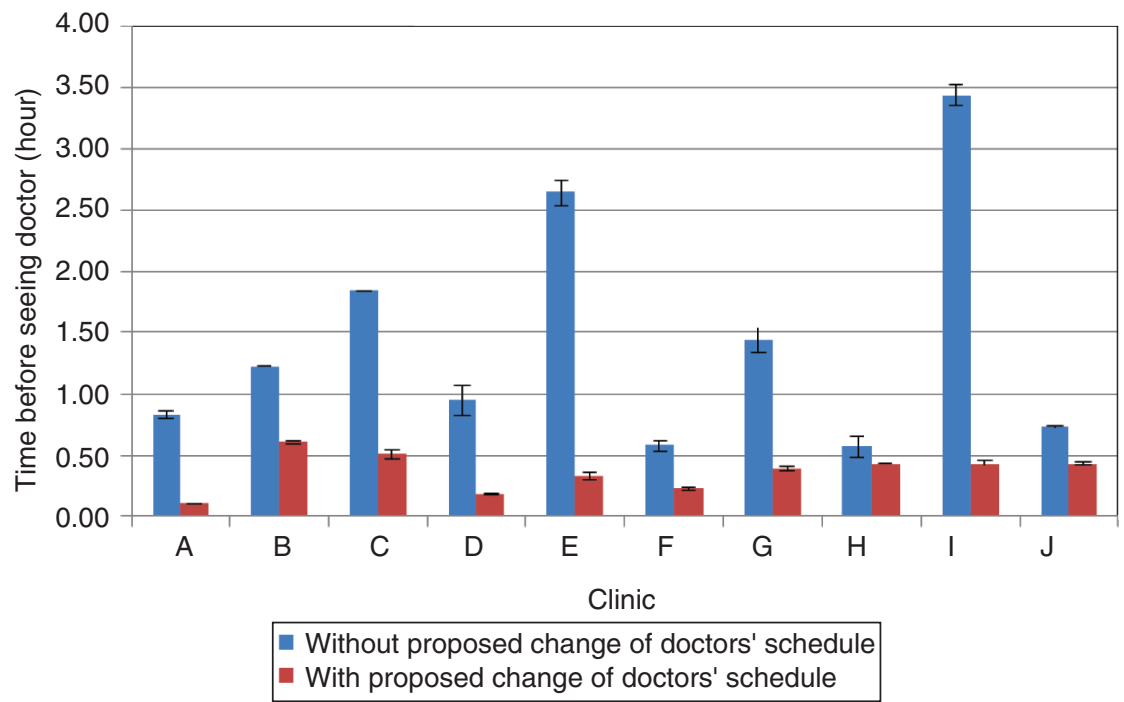

Figure 6. Comparison of average patient's time before seeing a doctor with and without proposed change of doctors' schedule.

with and without the proposed change of doctors' schedule. The data show that with the proposed change of doctors' schedule, such time is significantly reduced and more evenly distributed across all clinics.

\subsection{Prediction of Impacts of Patient Increase}

We hypothesize that when the orthopedic OPD wards move to a new and larger facility, the number of patients will increase. Under the current settings, we study how a $50 \%$ increase in patient arrival rates will impact the service performance (see Figure 7 and Table 6). In Figure 7, it is shown that 50\% increase of patients has a higher impact on the total time service level with the by-appointment and transferred patients, and a lower impact with the walk-in patients. The walk-in patients are already required to wait for any available open slots, resulting in a very low service level. In Table 6 , the overtime refers to the time that doctors have to beyond their scheduled working hours (see Table A2 for the current resource schedule). The clinics that operate in the evening, or the third shift, perform the best and can mostly complete services within one hour of overtime. The clinics that operate on Tuesday and Friday afternoons (Shift 2) are the most problematic. The morning shift of the Saturday clinic can experience some difficulties due to the limited numbers of doctors on duty, especially in case of higher demand.

\subsection{Further Discussion of the Results}

We have demonstrated how the DES model can be used as a decision-support tool for OPD management, especially for what-if analysis and resource capacity planning. This case study quantifies the problems of high congestion commonly encountered in large public hospitals in Thailand and other developing countries (e.g., in terms of waiting 


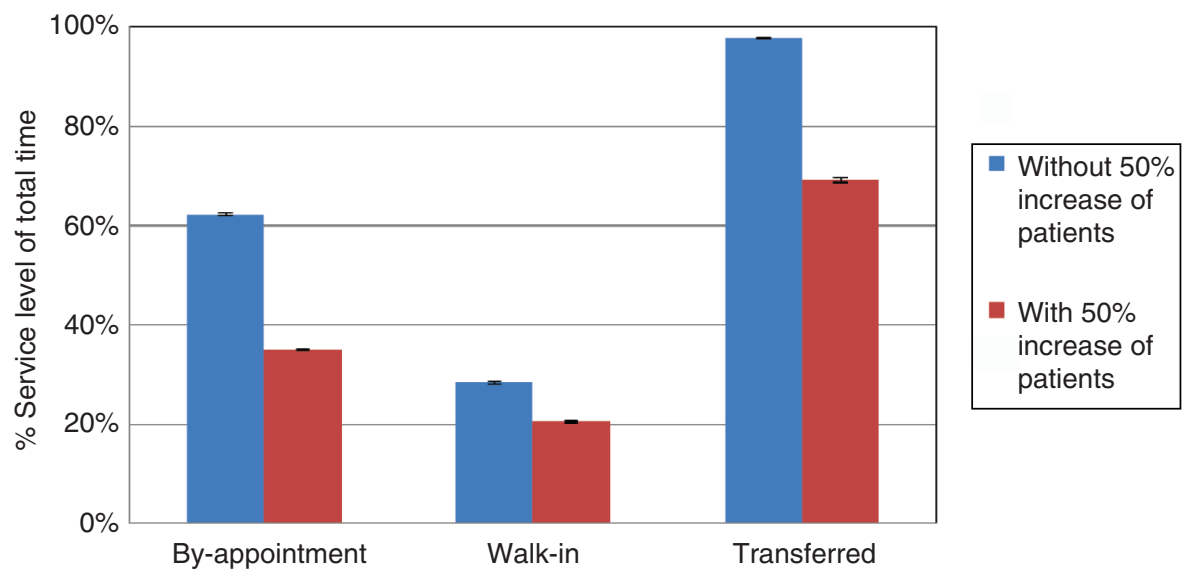

Figure 7. Comparison of service level of total time in system by patient types, as well as with and without $50 \%$ increase in number of patients.

Table 6. Comparison of doctors' overtime with and without $50 \%$ increase in the number of patients. All data in hours

\begin{tabular}{lcccccc}
\hline & \multicolumn{2}{c}{ Morning shift } & \multicolumn{2}{c}{ Afternoon shift } & \multicolumn{2}{c}{ Evening shift } \\
Day & Without $^{\mathbf{a}}$ & With $^{\mathbf{b}}$ & Without & With & Without & With \\
\hline Sunday & $0.68 \pm 0.06$ & $1.65 \pm 0.24$ & \multicolumn{2}{c}{ n/a } & \multicolumn{2}{c}{$\mathrm{n} / \mathrm{a}$} \\
Monday & $1.39 \pm 0.05$ & $2.50 \pm 0.21$ & $1.98 \pm 0.07$ & $3.56 \pm 0.22$ & $0.47 \pm 0.06$ & $0.67 \pm 0.20$ \\
Tuesday & $1.46 \pm 0.06$ & $2.44 \pm 0.23$ & $4.63 \pm 0.07$ & $7.15 \pm 0.18$ & $0.46 \pm 0.07$ & $1.05 \pm 0.24$ \\
Wednesday & $1.58 \pm 0.05$ & $2.32 \pm 0.11$ & $2.53 \pm 0.05$ & $4.41 \pm 0.20$ & $0.48 \pm 0.05$ & $0.79 \pm 0.12$ \\
Thursday & $1.30 \pm 0.05$ & $1.80 \pm 0.10$ & $1.73 \pm 0.06$ & $2.28 \pm 0.14$ & $0.21 \pm 0.06$ & $0.50 \pm 0.18$ \\
Friday & $1.47 \pm 0.04$ & $3.07 \pm 0.21$ & $5.66 \pm 0.07$ & $7.38 \pm 0.11$ & $0.56 \pm 0.06$ & $1.10 \pm 0.22$ \\
Saturday & $2.42 \pm 0.10$ & $6.49 \pm 0.28$ & $2.54 \pm 0.05$ & $4.28 \pm 0.14$ & \multicolumn{2}{c}{$\mathrm{n} / \mathrm{a}$} \\
\hline
\end{tabular}

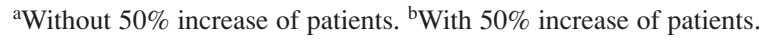

times, number of patients in the waiting areas, percentage of patients receiving services within a target time, doctor's utilization and doctor's overtime). Beyond adopting the lean process improvement, quantitative analysis using DES helps management correctly identify bottleneck processes and resource-critical clinics. The quality of service can be reflected through average patients' waiting time, time in system, and percentage of patients who can see their doctors within the target time. Quantitative information of congestion in the clinic can be obtained through doctors' utilization, patient-to-doctor ratio, and doctors' overtime. 
The most challenging aspect of implementing the proposed measures to improve the present system may be in convincing people to adopt new practices. This may sound simple, but requires careful prior design and preparation of the management process, since the hospital is people-centric and involves many stakeholders. The study hospital will begin implementing an IT medical records system as well as the doctor schedule straddling in some wards, especially within premium OPD services. It is common to see large, public hospitals pay more attention towards everyday OPD visits since it is more profitable compared with inpatient hospital services. Some well-known public hospitals have offered premium services at higher prices for more efficient services, such as shorter waiting time and quicker appointment turnaround for similar clinical procedures.

With regard to poor service, the hospital should be able to devote more efforts to improving service levels. When patients spend a long time in a hospital, it is not only time wasted for the patients, but also represent high indirect costs for the hospital due to inefficient service. These inefficiencies require hospitals to provide more seats and space for waiting patients and an increased risk of infection to the patients. Across different sectors of industry including healthcare, the concept of reduction or even elimination of no-added-value activities and process variation has been seriously considered as a means to improve efficiency.

\section{CONCLUSION}

This case study illustrates common problems encountered in large public hospitals in Thailand and perhaps in other developing countries. It confirms the fact of high patient demand in a large public hospital due to low costs of hospital services and highly qualified physicians despite low service levels. DES simulation model as a tool enables testing of different ideas and various scenarios before implementation. Our model is constructed with specific reference to an OPD in a large public hospital with high patient-to-doctor ratio. Future research may test the present framework on other OPD wards in large public hospitals in Thailand as well as other developing countries.

\section{ACKNOWLEDGEMENTS}

We would like to thank our reviewers and the editor for their careful reading, and constructive feedback. We would also like to thank the hospital staff members for their invaluable support to this research. This research is sponsored by the National Research University Grant to Mahidol University, given by the Commission of Higher Education, the Royal Thai Government.

\section{CONFLICT OF INTEREST}

The authors indicated no potential conflicts of interest.

\section{REFERENCES}

[1] Law AM, Simulation Modeling \& Analysis, 4th ed., McGraw-Hill, Inc., New York, 2007.

[2] Henderson SG, Nelson BL, Handbooks in Operations Research and Management Science: Simulation, Elsevier, Amsterdam, 2006. 
[3] Seila AF, Brailsford S, Opportunities and challenges in health care simulation. Advancing the Frontiers of Simulation: A Festschrift in Honor of George Samuel Fishman, International Series in Operations Research \& Management Science, Springer Science+Business Media, LLC, 2009:195-229.

[4] Weng ML, Houshmand AA, Healthcare simulation: a case study at a local clinic, in: Farrington PA, Nembhard H B, Sturrock DT, Evans GW (ed). Proceeding of the 1999 Winter Simulation Conference. 1999, 1577-1584.

[5] Raouf A, Ben-Daya M, Outpatient clinic's staffing: a case Study. International Journal of Health Care Quality, 1997, 10:229-232.

[6] Zhu ZC, Heng BH, Teow KL, Simulation study of the optimal appointment number for outpatient clinics. International Journal of Simulation Modelling, 2009, 8(3):156-165.

[7] Wijewickrama A, Takakuwa S, Simulation analysis of appointment scheduling in an outpatient department of internal medicine, in: Kuhl ME, Steiger NM, Armstrong FB, Joines JA (ed). Proceeding of the 2005 Winter Simulation Conference, 2005, 2264-2273.

[8] Su S, Shih C-L, Managing a mixed-registration-type appointment system in outpatient clinic. International Journal of Medical Informatics. 2003, 70:31-40.

[9] Ballard SM, Kuhl ME, The use of simulation to determine maximum capacity in the surgical suit operating room, in: Perrone LF, Wieland FP, Liu J, Lawson BG, Nicol DM, Fujimoto RM (ed). Proceeding of the 2006 Winter Simulation Conference, 2006, 433-438.

[10] Beasler FF, Jahnsen HE, DaCosta M, The use of simulation and design of experiments for estimating maximum capacity in an emergency room, in: Chick S, Sanchez PJ, Ferrin D, Morrice DJ (ed). Proceeding of the 2003 Winter Simulation Conference, 2003, 1903-1906.

[11] Werker G, Sauré A, French J, and Shechter S, The use of discrete-event simulation modelling to improve radiation therapy planning process. Radiotherapy and Oncology, 2009, 92:76-82.

[12] Duguay C, Chetouane F, Modeling and improving emergency department systems using discrete event simulation. Simulation, 2007, 83(4):311-320.

[13] Brenner S, Zeng Z, Liu Y, Wang J, Li J, Howard PK, Modeling and analysis of the emergency department at university of Kentucky Chandler Hospital using simulations. Journal of Emergency Nursing, 2010, 36(4):303-310.

[14] Raghavan VA, Venkatadri V, Kesavakumaran V, Wang S, Khasawneh M, Srihari K, Reengineering the cardiac catheterization lab processes: a lean approach. Journal of Healthcare Engineering, 2010, $1: 45-65$.

[15] Oddoye JP, Jones DF, Tamiz M, Schmidt P, Combining simulation and goal programming for healthcare planning in a medical assessment unit. European Journal of Operational Research, 2009, 193:250-261.

[16] Villamizar JR, Coelli FC, Pereira WCA, Almeida RMVR, Discrete-event computer simulation methods in the optimisation of a physiotherapy clinic. Physiotherapy, 2010, 97(1):71-77.

[17] Su Q, Yao X, Su P, Shi J, Zhu Y, Xue L, Hospital registration process reengineering using simulation method. Journal of Healthcare Engineering, 2010, 1:67-82.

[18] Kelton WD, Sadowski RP, Swets NB. Simulation with Arena, 5th ed., McGraw-Hill, Inc, New York, 2010.

[19] Banks J, Carson JS, Nelson BL, Nicol DM. Discrete-Event System Simulation, 5th ed., Prentice-Hall, 2009. 


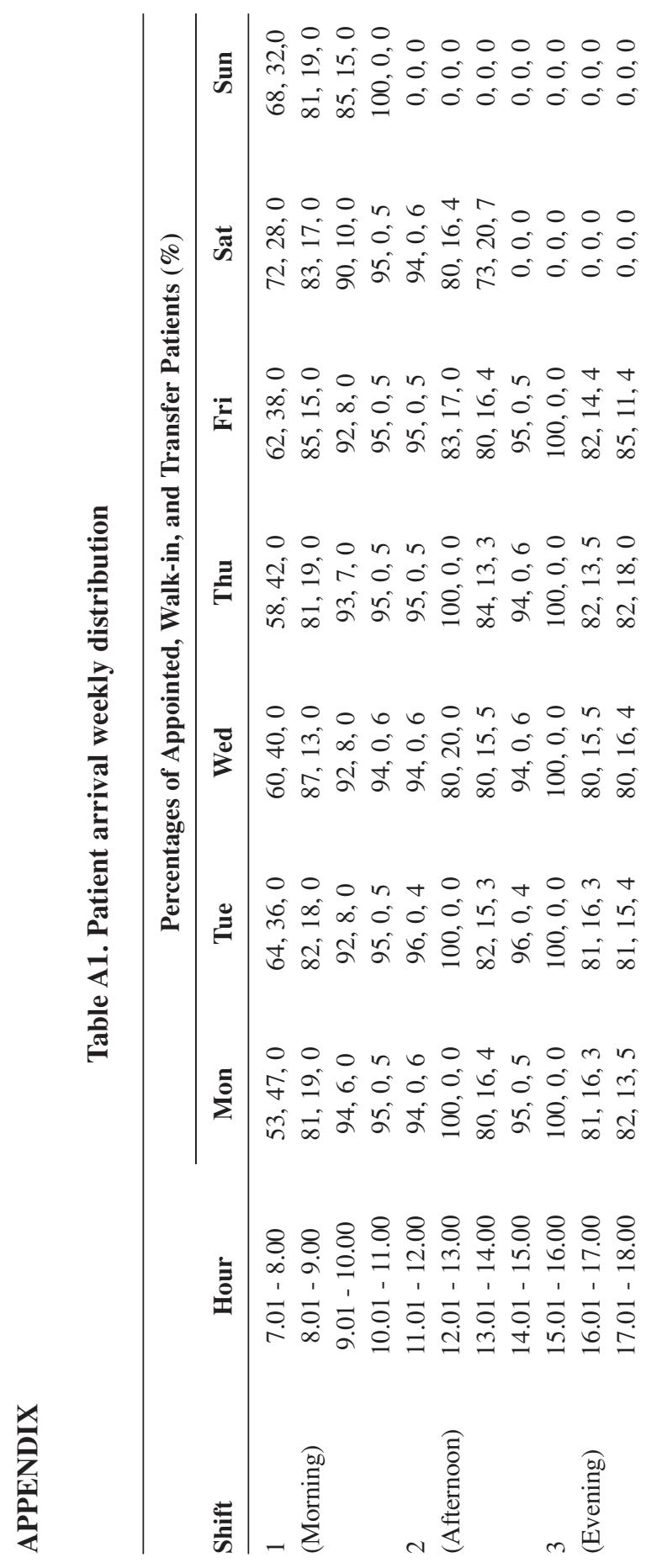


Table A2. Resource schedules for utilization calculation

\begin{tabular}{lcccccc}
\hline & \multicolumn{3}{c}{ Weekday } & & \multicolumn{2}{c}{ Weekend } \\
\cline { 2 - 4 } \cline { 6 - 7 } Resource type & Shift 1 & Shift 2 & Shift 3 & & Shift 1 & Shift 2 \\
\hline $\begin{array}{l}\text { Doctor/Pharmacy } \\
\text { Resident }\end{array}$ & $09.00-12.00$ & $13.00-15.00$ & $16.00-19.00$ & & $09.00-12.00$ & $13.00-15.00$ \\
Nurse/Clerk/ & $09.00-12.00$ & N/A & N/A & & N/A & N/A \\
Technician & $07.00-12.00$ & $13.00-15.00$ & $16.00-19.00$ & & $07.00-12.00$ & $13.00-15.00$
\end{tabular}

Table A3. Numbers of available doctors at the current orthopedic OPD

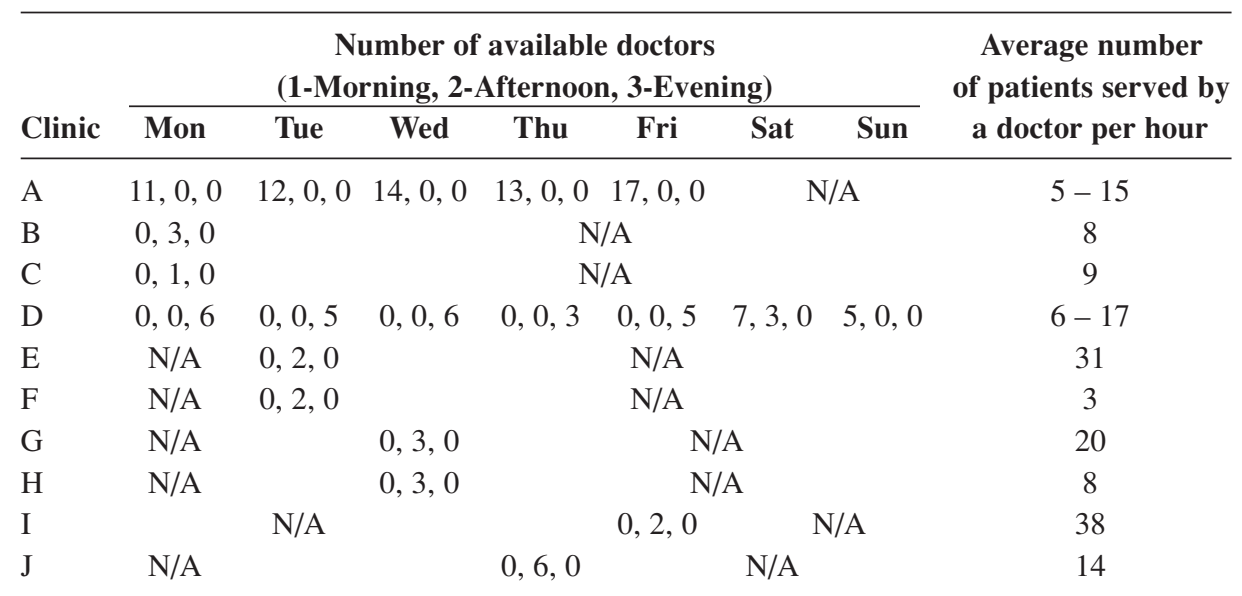


Table A4. Numbers of available doctors at the orthopedic OPD for the straddled schedule

\begin{tabular}{lccc}
\hline & \multicolumn{3}{c}{ Shift $\mathbf{1}$ - Morning (M, Tu, W, Th, F, Sa, Su) } \\
\cline { 2 - 4 } Clinic & $\mathbf{7 . 0 0 - \mathbf { 1 0 . 0 0 }}$ & $\mathbf{8 . 0 0 - \mathbf { 1 1 . 0 0 }}$ & $\mathbf{9 . 0 0 - \mathbf { 1 2 . 0 0 }}$ \\
\hline A & $4,4,5,5,6,0,0$ & $4,4,5,4,6,0,0$ & $3,4,4,4,5,0,0$ \\
D & $0,0,0,0,0,3,2$ & $0,0,0,0,0,2,2$ & $0,0,0,0,0,2,1$ \\
\hline
\end{tabular}

Shift 3 - Evening (M, Tu, W, Th, F, Sa, Su)

\begin{tabular}{lccc}
\cline { 2 - 4 } Clinic & $\mathbf{1 5 . 0 0 - \mathbf { 1 7 . 0 0 }}$ & $\mathbf{1 6 . 0 0 - \mathbf { 1 8 . 0 0 }}$ & $\mathbf{1 7 . 0 0 - \mathbf { 1 9 . 0 0 }}$ \\
\hline $\mathrm{D}$ & $2,2,2,1,2,0,0$ & $2,2,2,1,2,0,0$ & $2,1,2,1,1,0,0$
\end{tabular}

Shift 2 - Afternoon

(M, Tu, W, Th, F, Sa, Su)

\begin{tabular}{lcc}
\cline { 2 - 3 } Clinic & $\mathbf{1 5 . 0 0 - \mathbf { 1 7 . 0 0 }}$ & $\mathbf{1 6 . 0 0 - \mathbf { 1 8 . 0 0 }}$ \\
\hline $\mathrm{B}$ & $2,0,0,0,0,0,0$ & $1,0,0,0,0,0,0$ \\
$\mathrm{C}$ & $\mathrm{N} / \mathrm{A}$ & $1,0,0,0,0,0,0$ \\
$\mathrm{D}$ & $0,0,0,0,0,2,0$ & $0,0,0,0,0,1,0$ \\
$\mathrm{E}$ & $0,1,0,0,0,0,0$ & $0,1,0,0,0,0,0$ \\
$\mathrm{~F}$ & $0,1,0,0,0,0,0$ & $0,1,0,0,0,0,0$ \\
$\mathrm{G}$ & $0,0,2,0,0,0,0$ & $0,0,1,0,0,0,0$ \\
$\mathrm{H}$ & $0,0,2,0,0,0,0$ & $0,0,1,0,0,0,0$ \\
I & $0,0,0,0,1,0,0$ & $0,0,0,0,1,0,0$ \\
$\mathrm{~J}$ & $0,0,0,3,0,0,0$ & $0,0,0,3,0,0,0$
\end{tabular}





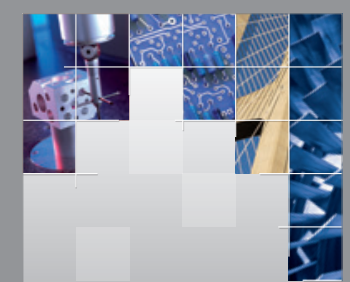

\section{Enfincering}
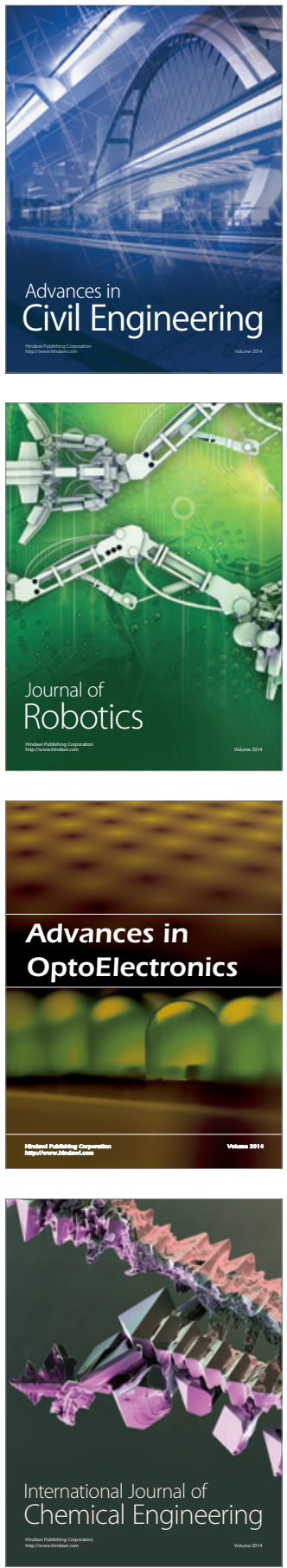

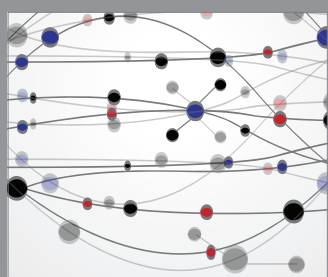

The Scientific World Journal

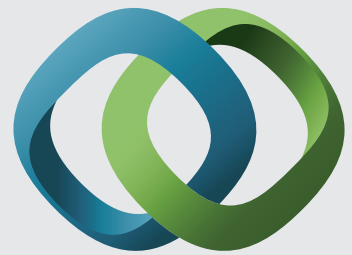

\section{Hindawi}

Submit your manuscripts at

http://www.hindawi.com
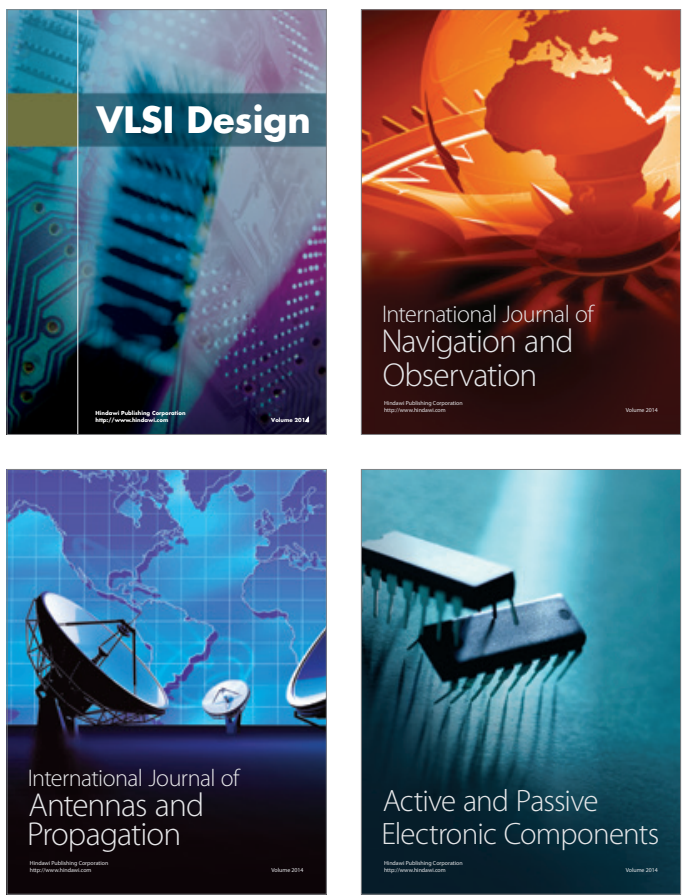
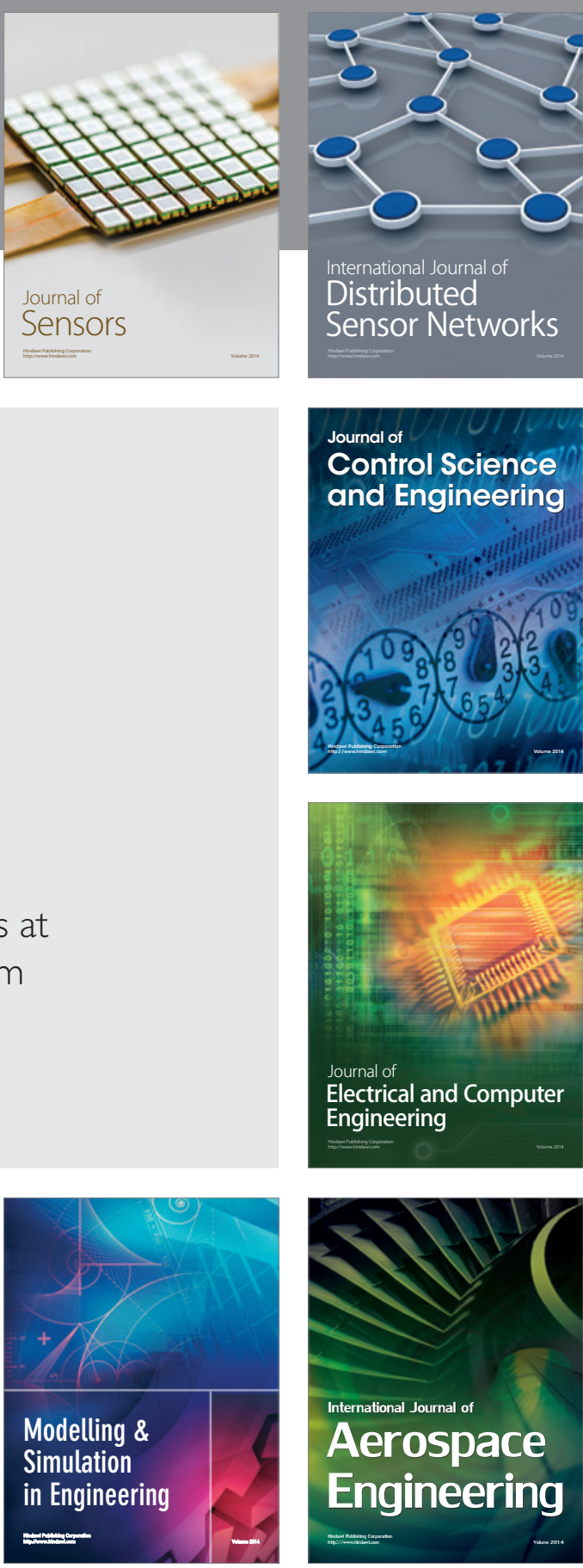

International Journal of

Distributed

Sensor Networks

Journal of

Control Science

and Engineering
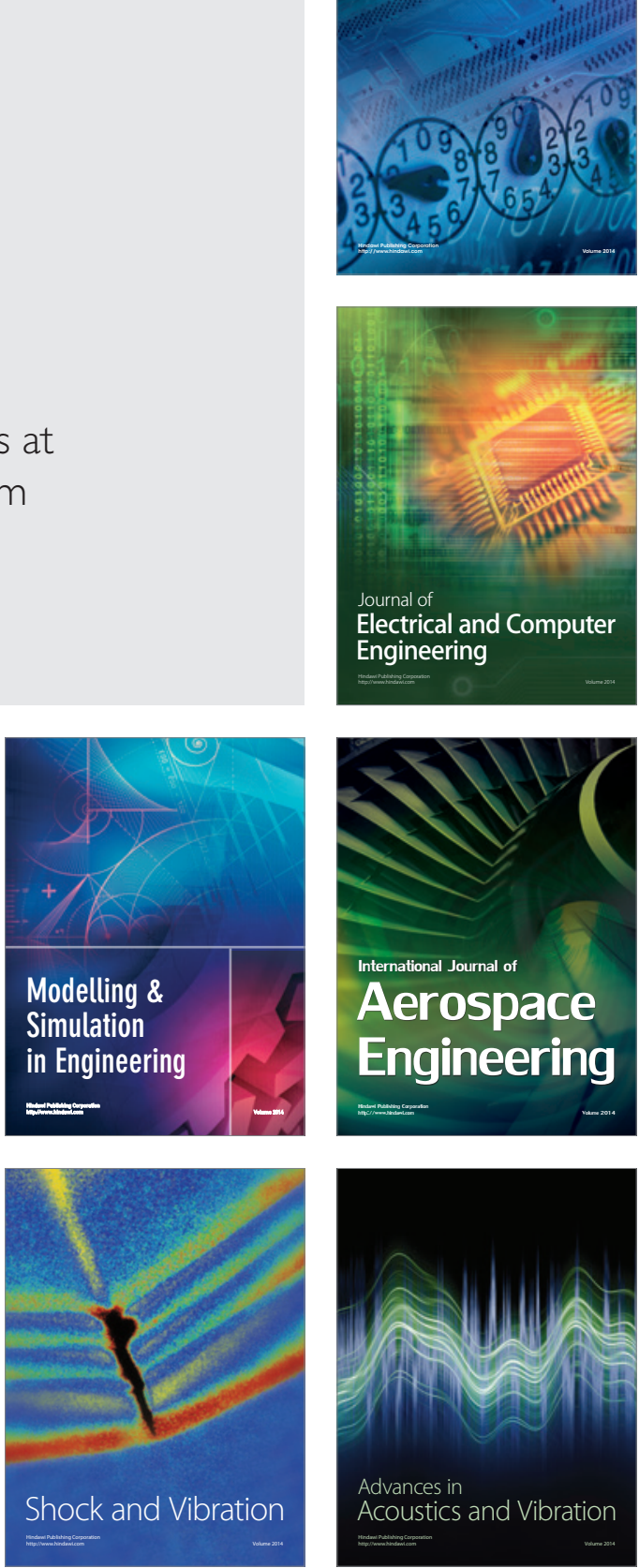